\title{
Coeficientes de escorrentía instantáneos para la cuenca del río Tutuvén, VII Región del Maule, Chile
}

\author{
Instantaneous runoff coefficients for Tutuvén river basin, Maule Region, Chile
}

\author{
Roberto Pizarro Tapia ${ }^{1 *}$, Marcela Tapia Cornejo ${ }^{1}$, Leonardo Román Arellano1, \\ Cristián Jordán Díaz ${ }^{1}$, César Farías Daza ${ }^{1}$ \\ *Autor de correspondencia: ${ }^{1}$ Universidad de Talca, Facultad de Ciencias Forestales, \\ Departamento de Gestión Forestal y Ambiental, Avenida Lircay s/n, Talca, Tel.: 71-200375, \\ rpizarro@utalca.cl
}

\begin{abstract}
SUMMARY
In Tutuvén river basin, central Chile, instantaneous runoff coefficient $(\mathrm{C})$, defined as the rate between runoff and rainfall, and its influence on different vegetation cover considering previous soil moisture were studied. Coefficients were calculated for intervals of 1, 2 and 4 hours in three different vegetation covers and three different moistures soil conditions, defined by the Curve Number method. 30 storms were used for the period 1982-1997. Results showed that the highest runoff coefficient values were obtained for one hour, with high moisture in soil and thin cover vegetation. On the other hand, the lowest runoff coefficient values were observed in sites of high cover vegetation and dry soils. It is concluded that rainfall intensity is the main factor influencing runoff coefficient values. Good estimates of $\mathrm{C}$ were obtained through a regression analysis between daily rainfall amount and $\mathrm{C}$, with a $\mathrm{R}^{2}$ ranging from 0.84 to 0.97 .
\end{abstract}

Key words: runoff coefficient, curve number method, rainfall intensity, rainfall-runoff relationship.

\section{RESUMEN}

En la cuenca del río Tutuvén, VII Región de Chile, se estudió el comportamiento del coeficiente de escorrentía instantáneo (C), definido como la relación entre las precipitaciones efectivas y las precipitaciones totales caídas, y la influencia sobre éste de diversas situaciones de vegetación y condiciones precedentes de humedad del suelo. Los coeficientes se determinaron para duraciones de 1, 2 y 4 horas, asociados a tres coberturas de vegetación y a las tres condiciones precedentes de humedad señaladas por el método, para 30 tormentas del periodo 1982-1997. Según los resultados obtenidos, los C mostraron valores mayores para la duración de una hora, y con vegetación escasa y condición de humedad alta. Por el contrario, los valores más bajos fueron para las situaciones con vegetación plena y condiciones secas de humedad del suelo. De todas las situaciones estudiadas, la intensidad de precipitación fue el factor más influyente en la determinación del coeficiente de escorrentía. Por otra parte, se obtuvieron buenas estimaciones de $\mathrm{C}$ a partir de las precipitaciones totales en 24 horas, presentando valores de $\mathrm{R}^{2}$ entre 0,84 y 0,97 .

Palabras clave: coeficiente de escorrentía, método del número de curva, intensidad de precipitación.

\section{INTRODUCCIÓN}

La cubierta vegetal cumple un rol elemental en cuanto a la disponibilidad de agua y los caudales generados por las precipitaciones (López 1998). Los efectos que provoca la vegetación son diversos, principalmente por los cambios producidos en la modificación y/o remoción de su cobertura. Una reducción de la cubierta de bosques genera alteraciones al ciclo hidrológico, como también una redistribución de las precipitaciones y un aumento en la esco- rrentía superficial y en los procesos erosivos (Reinhart et al. 1963, Wright et al. 1990), ya que la vegetación actúa como un factor interceptor y regulador de dichos procesos (Meunier 1996). Por otra parte, la presencia de cobertura vegetal, principalmente masas forestales, provoca cantidades importantes de intercepción de las precipitaciones (Huber y Oyarzún 1983, Oyarzún et al. 1985), así como también rebajas en los caudales (Calder 1992, Iroumé y Huber 2000), aunque la magnitud de respuesta es altamente variable e impredecible (Hibbert 1996). 
En este contexto, es importante e interesante el estudio de los cambios de cobertura vegetal y las alteraciones que ésta provoca, sobre todo, en cuencas que no poseen control fluviométrico en su salida. Para esto es necesario contar con métodos que permitan inferir comportamientos de las escorrentías a partir de otras variables. Uno de los métodos más utilizados es el del "número de curva", propuesto por el USSCS (López 1998), el cual permite estimar escorrentías directas o precipitaciones efectivas a partir de información de precipitaciones (Gujarati 1992, Chow et al. 1994, López 1998). Con la información generada por la aplicación de este tipo de metodologías, se pueden obtener relaciones entre las precipitaciones y las precipitaciones efectivas, determinando de esta forma tasas y/o coeficientes de escurrimiento, lo que permite determinar porcentajes de escurrimiento, según sean los montos e intensidades de precipitación.

En el presente trabajo, se determinan y analizan coeficientes de escorrentía instantáneos, determinados a base del método del número de curva, para la cuenca del río Tutuvén, VII Región de Chile. Ello, con la finalidad de determinar mediante simulaciones de cobertura vegetal, la influencia de ésta en las relaciones entre la precipitación efectiva y la precipitación total.

\section{MÉTODOS}

Área de estudio. La cuenca del río Tutuvén se ubica en la provincia de Cauquenes, VII Región de Chile $\left(35^{\circ} 41^{\prime}\right.$ y $35^{\circ} 55^{\prime}$ 'S y $72^{\circ} 08^{\prime}$ y $72^{\circ} 26^{\prime} \mathrm{O}$ ). Pertenece a la cuenca hidrográfica del río Loncomilla, dentro de la hoya hidrográfica del río Maule y cuenta con una superficie de $211,78 \mathrm{~km}^{2}$ (figura 1). La cuenca posee un clima templado mediterráneo, con diferencias en sentido Norte-Sur, caracterizado por una estación seca de cuatro meses. Posee precipitaciones medias anuales de $816 \mathrm{~mm}$. En cuanto a la temperatura, los promedios anuales fluctúan dependiendo de la ubicación, la influencia marina y sus características orográficas. La temperatura media anual oscila entre $13^{\circ} \mathrm{C}$ y $15^{\circ} \mathrm{C}$, con extremas de $22^{\circ} \mathrm{C}$ durante el periodo de verano; en cambio, en invierno las temperaturas mínimas medias son de $6^{\circ} \mathrm{C}$ (Dirección General de Aguas 1987). La vegetación predominante en la cuenca está conformada por praderas y matorrales, y especies arbóreas, tales como boldo (Peumus boldus Mol.), litre (Lithraea caustica Hook. et Arn.), espino (Acacia caven [Mol.] Mol.), maqui (Aristotelia chilensis [Mol.] Stuntz.), lingue (Persea lingue Nees), peumo (Cryptocarya alba [Mol.] Looser), quillay (Quillaja saponaria Mol.), olivillo (Aextoxicon punctatum R. et Pav.), canelo (Drimys winteri J. R. et Forster), arrayán (Luma apiculata [DC.] Burret), roble (Nothofagus obliqua [Mirb.] Oerst.), hualo (Nothofagus glauca [Phil.] Krasser) y ruil (Nothofagus alessandrii Espinosa); además de especies exóticas con fines productivos, como pino insigne (Pinus radiata D. Don), y variedades de eucalipto (Eucalyptus sp.) y álamo (Populus sp.), entre otras (CONAF-CONAMA 1987).

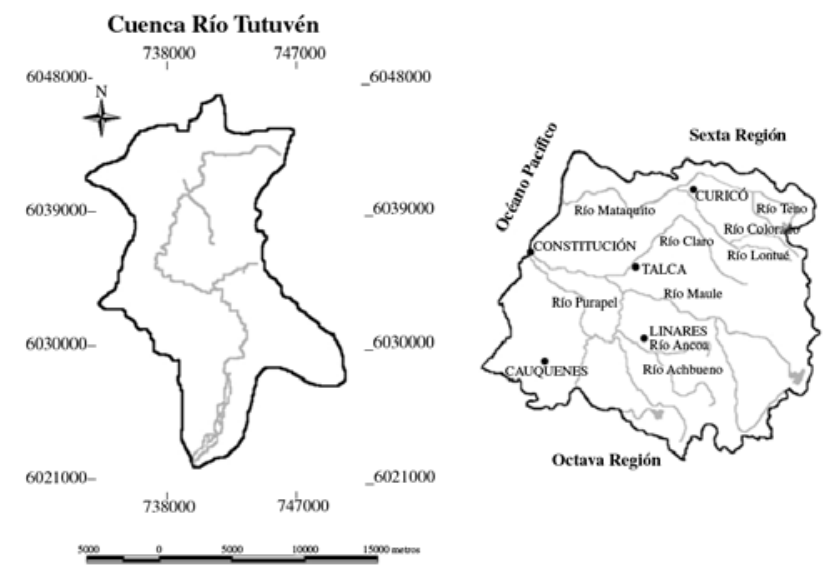

Figura 1. Ubicación de la cuenca del río Tutuvén. Location of Tutuvén river basin.

Selección de tormentas. La cuenca del río Tutuvén no posee estaciones pluviográficas, por lo que se utilizó la estación Melozal ( $35^{\circ} 44^{\prime} \mathrm{S}, 71^{\circ} 48^{\prime} \mathrm{O}$ ), sobre la cual se realizó la selección de tormentas, debido a su cercanía a la cuenca y similar régimen de precipitaciones. Para ello se analizaron las bandas de registro del pluviógrafo y se seleccionaron 30 tormentas, 15 de alta intensidad y 15 de baja intensidad, entre los meses de mayo y agosto (periodo de mayor pluviometría en la zona), para los años 1982 y 1997. La elección de las tormentas se hizo de manera visual, seleccionando aquellas que tuvieran un registro continuo de precipitación en 24 horas, tomando como referencia las 08:00 h de un día, hasta las 08:00 h del día siguiente, según el criterio de medición de las precipitaciones de la Dirección General de Aguas (D.G.A.). Las tormentas de alta intensidad presentaron en el pluviograma un registro ascendente y abrupto en un lapso corto. Para el caso de las tormentas de baja intensidad se eligieron aquellas que presentaron una curva menos pronunciada, que denotaba la presencia de tormentas calmas.

Para cada evento seleccionado se midieron sobre el pluviograma los montos de precipitaciones para intervalos de 1, 2 y 4 horas. El cuociente entre estas alturas de agua y su correspondiente duración determinó las intensidades de precipitación $(\mathrm{mm} / \mathrm{h})$. En el cuadro 1 se presentan los montos de precipitación de las tormentas utilizadas.

Caracterización de la cuenca y determinación de escenarios de vegetación. Se caracterizó la cuenca en relación a los suelos y vegetación presentes. Para ello se utilizaron cartas topográficas y mosaicos, así como el catastro de los recursos vegetacionales (CONAF-CONAMA 1997). Las series de suelos determinadas fueron interpretadas mediante el manual Materiales y Símbolos 
Cuadro 1. Precipitación (Pp) total de las tormentas utilizadas en la determinación del coeficiente de escorrentía.

Total rainfall for each storm used to calculate runoff coefficient.

\begin{tabular}{cccrcc}
\hline Tormenta & Pp Total & Tormenta & Pp Total & Tormenta & Pp Total \\
\hline 1 & 62,0 & 11 & 56,2 & 21 & 22,0 \\
2 & 76,3 & 12 & 69,4 & 22 & 15,0 \\
3 & 62,9 & 13 & 132,4 & 23 & 23,1 \\
4 & 70,1 & 14 & 77,1 & 24 & 16,1 \\
5 & 87,6 & 15 & 52,3 & 25 & 24,1 \\
6 & 63,5 & 16 & 5,9 & 26 & 10,1 \\
7 & 74,5 & 17 & 9,0 & 27 & 15,1 \\
8 & 71,6 & 18 & 12,0 & 28 & 11,4 \\
9 & 48,2 & 19 & 11,3 & 29 & 7,5 \\
10 & 50,5 & 20 & 11,8 & 30 & 8,9 \\
\hline
\end{tabular}

1-15: Tormentas de alta intensidad. 16-30: Tormentas de baja intensidad.

(CIREN-CORFO 1983). De esta forma, se establecieron las clases y usos de los suelos y aspectos relacionados con la geomorfología del lugar, además de elementos como el desarrollo del perfil de suelo, el drenaje y la textura. En cuanto a la vegetación, la información base se obtuvo en formato digital desde el catastro de los recursos vegetacionales, a partir del cual se identificaron y clasificaron las diversas coberturas del sector.

La información de suelos y vegetación obtenida fue asimilada a los requerimientos del método del número de curva, asociándola a los cuatro grupos de suelos y a los tipos de vegetación que presenta el método. Con respecto a la vegetación, ésta se asoció a las tablas desarrolladas por Ponce (1989). Con ello se determinó el escenario actual de vegetación (VA), estableciéndose dos escenarios adicionales: un escenario de vegetación plena (VP) y uno de vegetación rala (VR). Al asociar estos tres escenarios a los tres tipos de condiciones precedentes de humedad que contempla el método, se determinaron nueve posibles escenarios hidrológicos para analizar.

Determinación de los números de curva. Luego de definidos los escenarios suelo-vegetación, se procedió a determinar los números de curva $(\mathrm{N})$, a través de las tablas de Ponce (1989). Para ello se tomó en cuenta que el número de curva tuviera relación con la mayor o menor escorrentía provocada según el tipo de vegetación. Posteriormente se calculó el parámetro $\mathrm{S}$ [1] que corresponde a la máxima infiltración del suelo, y el umbral de escorrentía I $\mathrm{I}_{\mathrm{o}}$ [2] (López 1998):

$$
\begin{gathered}
S=254 *\left(\frac{100}{N}-1\right) \\
I_{o}=0,2 * S
\end{gathered}
$$

Con esta información se determinaron las precipitaciones efectivas según las ecuaciones [3] y [4]:

$$
Q_{i}=0 \quad \text { Si } \Sigma \mathrm{P} \leq 0,2 \mathrm{~S}
$$

$$
Q_{i}=\frac{\left(\sum P_{j}-0,2 * S\right)^{2}}{\left(\sum P_{j}+0,8 * S\right)}-Q_{(I-l)} \quad \mathrm{Si} \sum \mathrm{P}>0,2 \mathrm{~S}
$$

Coeficiente de escorrentía. Posteriormente se determinaron los coeficientes de escorrentía instantáneos para 1,2 y 4 horas, y para las distintas situaciones planteadas [5]. Estos se determinaron de manera discreta, por medio del cuociente de las diferencias de precipitaciones efectivas y de precipitaciones (CONAF-CONAMA 1997):

$$
C_{i j}=\frac{\left(P e_{i j}-P e_{i(j-I)}\right)}{\left(P_{i j}-P_{i(j-I)}\right)}
$$

donde $\mathrm{C}_{\mathrm{ij}}, \mathrm{Pe}_{\mathrm{ij}}$ y $\mathrm{P}_{\mathrm{ij}}$ representan, respectivamente, el coeficiente de escorrentía, la precipitación efectiva y la precipitación para la tormenta i, durante la hora j, con Pe y $P$ en milímetros, y i(j-1) es el intervalo inmediatamente anterior.

Estimación de C a partir de precipitaciones. Considerando los nueve escenarios planteados, se procedió a establecer regresiones lineales entre los montos de precipitación y los coeficientes de escorrentía máximos de cada tormenta, tanto de alta como de baja intensidad, de manera de establecer funciones que representen la relación entre estas variables. Para ello, las funciones obtenidas fueron sometidas a algunos supuestos del método de mínimos cuadrados como normalidad, homocedasticidad y autocorrelación (Cid et al. 1990, Gujarati 1992, Montgómery et al. 1996). La calidad del ajuste se evaluó a través del coeficiente de determinación $\left(\mathrm{R}^{2}\right)$ y el error estándar de estimación (EEE).

\section{RESULTADOS}

Caracterización de la cuenca. Según la interpretación de la información entregada por los mosaicos y cartas, la cuenca presentó suelos con capacidad de drenaje buenas a moderadas; y texturas franco arenosas y franco arcillosas. Ambos conceptos, en conjunto, determinaron los siete tipos de suelos existentes en la zona, los cuales se asimilaron a los suelos de los grupos $\mathrm{B}$ y $\mathrm{C}$, según el método del número de curva (figura 2).

En cuanto a la vegetación existente, según los resultados arrojados por la interpretación del catastro, en la 

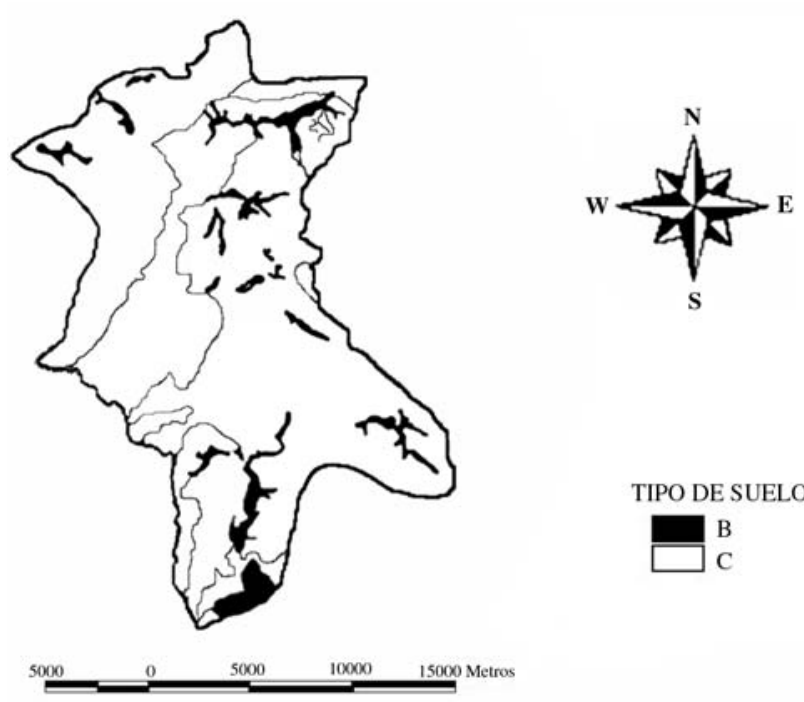

Figura 2. Grupos de suelos generados para la cuenca del Tutuvén.

Soil groups generated for Tutuvén river basin.

cuenca existen 16 coberturas vegetales (cuadro 2). Los matorrales y matorrales arborescentes fueron seleccionados dentro del tipo matorral. Los bosques nativos, exóticos y las plantaciones forestales fueron clasificados dentro del tipo de cobertura de bosques, determinados principalmente por tener una mayor cobertura de intercepción y mayor capacidad de infiltración; por ende, les cabe la asignación de un menor número de curva representativo de la escorrentía.

Las praderas anuales, rotación cultivo-pradera y los terrenos de uso agrícola fueron clasificados bajo el tipo grano pequeño, sembrado en línea o pradera de rotación (hileras rectas $[\mathrm{SR}]$ ) y cultivos densos de leguminosas, respectivamente. Estos usos del suelo presentan una baja cobertura de intercepción, la que favorece las escorrentías superficiales.

$\mathrm{Al}$ asociar las coberturas vegetales con los grupos de suelos se produjeron 17 combinaciones suelo-vegetación, las que determinaron todos los posibles números de curva a determinar (figura 3 ).

Número de curva. El valor del número de curva (NC), y de los parámetros $\mathrm{S}$ y $\mathrm{I}_{\mathrm{o}}$, para los distintos escenarios asociados y para las tres condiciones de humedad se presentan en el cuadro 3. En él se aprecia que de los nueve escenarios, los que presentaron mayores números de curva fueron aquellos que se encontraron bajo la condición de humedad III. Dentro de estos, el noveno escenario (VRCIII) fue el que registró el mayor valor $(91,85)$; a su vez, este resultado provocó los mínimos valores para los parámetros $\mathrm{S}_{\mathrm{S}} \mathrm{I}_{\mathrm{o}}$, con $22,54 \mathrm{~mm}$ y $4,51 \mathrm{~mm}$, respectivamente. Esto determinó una situación de mínima vegetación en la condición de máxima humedad, es decir, de suelo saturado, lo que definió que todas las tormentas generaran escorrentía superficial.

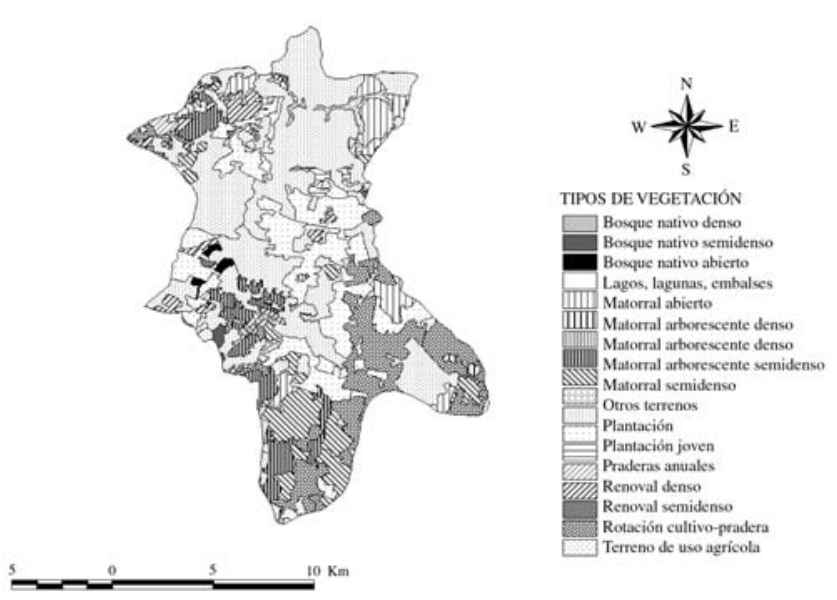

Figura 3. Detalle de la base de datos de la intersección entre grupos de suelo y la vegetación para la cuenca del Tutuvén.

Database details of the intersection between soils groups and vegetal cover for Tutuven basin.

Por otra parte, los menores NC fueron estimados para la condición de humedad I, siendo el escenario (VPCI), el que registró el valor menor: 39,83. Esta situación determinó el mayor valor de los parámetros $\mathrm{S}$ e $\mathrm{I}_{0}$, con $383,71 \mathrm{~mm}$ y $76,74 \mathrm{~mm}$, respectivamente. La magnitud del umbral crítico de escorrentía provocó que sólo dos tormentas seleccionadas, 5 y 13 , superaran este valor y generaran escorrentía.

De las 30 tormentas analizadas destacan las tormentas 5 y 13, con los mayores montos e intensidades de precipitación. En la figura 4 se aprecia una tendencia al aumento en el comportamiento de las escorrentías, a medida que las precipitaciones aumentan. Por otro lado, y según lo señalado anteriormente, es en la condición de mayor humedad (III) donde se producen las mayores escorrentías y se establece la superación más temprana del parámetro $\mathrm{I}_{\mathrm{o}}$.

Coeficiente de escorrentía. En los cuadros 4 y 5 se presentan los coeficientes de escorrentía promedios determinados para los nueve escenarios y distintas duraciones planteadas. Éstas muestran la gran variabilidad que alcanza el coeficiente de escorrentía para cada lapso, y esto derivado de las distintas condiciones de humedad del suelo. Asimismo, se verificó que para la duración de una hora, se obtuvieron los valores más altos y con diferencias notorias con respecto a las otras duraciones, que mostraron intensidades menores. A pesar de esto, en las tormentas de alta intensidad y para la duración de dos horas, en algunos casos se presentaron valores similares que para la duración de una hora, reflejando el alto nivel de intensidades registradas. En el caso de las tormentas de baja intensidad, la obtención de un número reducido de coeficientes, y de bajos valores de estos, se debe a la 
Cuadro 2. Cubiertas vegetales y números de curva determinados para la vegetación actual.

Type of vegetation cover and curve numbers calculated.

\begin{tabular}{|c|c|c|c|c|}
\hline $\begin{array}{l}\text { Cubierta actual } \\
\text { de la zona }\end{array}$ & $\begin{array}{l}\text { Clasificación de vegetación } \\
\text { según el Número de Curva }\end{array}$ & $\begin{array}{l}\text { Estado } \\
\text { hidrológico }\end{array}$ & $\begin{array}{r}\mathrm{N}^{\circ} \mathrm{C} \\
\text { Grup } \\
\mathrm{B}\end{array}$ & $\begin{array}{l}\text { según } \\
\text { Suelo } \\
\text { C }\end{array}$ \\
\hline $\begin{array}{l}\text { Bosque nativo } \\
\text { denso }\end{array}$ & Bosques & Bueno & 55 & 70 \\
\hline $\begin{array}{l}\text { Bosque nativo } \\
\text { semidenso }\end{array}$ & $\begin{array}{l}\text { Matorral, mezcla de matorral y maleza siendo } \\
\text { el matorral el elemento prioritario }\end{array}$ & Medio & 56 & 70 \\
\hline Matorral abierto & $\begin{array}{l}\text { Matorral, mezcla de matorral y maleza siendo } \\
\text { el matorral el elemento prioritario }\end{array}$ & Malo & 67 & 77 \\
\hline $\begin{array}{l}\text { Matorral } \\
\text { semidenso }\end{array}$ & $\begin{array}{l}\text { Matorral, mezcla de matorral y maleza siendo } \\
\text { el matorral el elemento prioritario }\end{array}$ & Medio & 56 & 70 \\
\hline $\begin{array}{l}\text { Matorral } \\
\text { arborescente } \\
\text { abierto }\end{array}$ & $\begin{array}{l}\text { Matorral, mezcla de matorral y maleza siendo } \\
\text { el matorral el elemento prioritario }\end{array}$ & Malo & 67 & 77 \\
\hline $\begin{array}{l}\text { Matorral } \\
\text { arborescente } \\
\text { semidenso }\end{array}$ & $\begin{array}{l}\text { Matorral, mezcla de matorral y maleza siendo } \\
\text { el matorral el elemento prioritario }\end{array}$ & Medio & 56 & 70 \\
\hline $\begin{array}{l}\text { Matorral } \\
\text { arborescente denso }\end{array}$ & $\begin{array}{l}\text { Matorral, mezcla de matorral y maleza siendo } \\
\text { el matorral el elemento prioritario }\end{array}$ & Bueno & 48 & 65 \\
\hline Plantación & Bosques & Bueno & 55 & 70 \\
\hline Plantación joven & Bosques & Media & 60 & 73 \\
\hline Praderas anuales & Grano pequeño (SR) & Buena & 75 & 83 \\
\hline Renoval abierto & $\begin{array}{l}\text { Matorral, mezcla de matorral y maleza siendo } \\
\text { el matorral el elemento prioritario }\end{array}$ & Malo & 67 & 77 \\
\hline Renoval semidenso & $\begin{array}{l}\text { Matorral, mezcla de matorral y maleza siendo } \\
\text { el matorral el elemento prioritario }\end{array}$ & Medio & 56 & 70 \\
\hline Renoval denso & $\begin{array}{l}\text { Matorral, mezcla de matorral y maleza siendo } \\
\text { el matorral el elemento prioritario }\end{array}$ & Bueno & 48 & 65 \\
\hline $\begin{array}{l}\text { Rotación cultivo- } \\
\text { pradera }\end{array}$ & $\begin{array}{l}\text { Sembrado en línea o pradera de rotación } \\
\text { (SR) }\end{array}$ & Mala & 77 & 85 \\
\hline $\begin{array}{l}\text { Terrenos de uso } \\
\text { agrícola }\end{array}$ & Cultivos densos de leguminosas $\mathrm{R}$ & Pobres & 77 & 84 \\
\hline
\end{tabular}

presencia de precipitaciones efectivas muy bajas con respecto a las precipitaciones totales caídas, incluso en la condición de humedad III.

Determinación de C por medio de precipitaciones. Durante el desarrollo de este proceso se estudiaron diversas variables y relaciones con el fin de determinar el coeficiente de escorrentía instantáneo a partir de precipitaciones. La variable precipitación en 24 horas fue la que presentó las relaciones más adecuadas. En el cuadro 6 se muestran los modelos seleccionados para la estimación del coeficiente de escorrentía. Los valores de $\mathrm{R}^{2}$ para los diversos escenarios fluctuaron en un rango de un $84,33 \%$ como mínimo para el octavo escenario (VRCII) y un máximo de $98,19 \%$, en el sexto escenario (VACIII). Además, el rango de valores del EEE fluctuó entre 0,028 como mínimo para el séptimo escenario (VRCI) y 0,149 como máximo para el octavo escenario (VRCII). Estos valores y el $\mathrm{R}^{2}$ definen la obtención de buenas estimaciones para el coeficiente de escorrentía. 
Cuadro 3. Resumen de los números de curva y el valor de $\mathrm{S}$ e $\mathrm{I}_{\mathrm{o}}$ para los nueve escenarios. Summary of curve numbers and $\mathrm{S}$ value and Io value for 9 situations studied.

\begin{tabular}{|c|c|c|c|c|c|c|c|c|c|}
\hline \multirow{3}{*}{ Vegetación } & \multicolumn{9}{|c|}{ Condición del estado de humedad previa } \\
\hline & & I & & & II & & & & \\
\hline & $\mathrm{NC}$ & $\mathrm{S}(\mathrm{mm})$ & $I_{o}$ & $\mathrm{NC}$ & $\mathrm{S}(\mathrm{mm})$ & $\mathrm{I}_{\mathrm{o}}$ & $\mathrm{NC}$ & $\mathrm{S}(\mathrm{mm})$ & $\mathrm{I}_{\mathrm{o}}$ \\
\hline Plena (VP) & 39,83 & 383,71 & 76,74 & 59,83 & 170,54 & 34,17 & 78,86 & 68,09 & 13,62 \\
\hline Actual (VA) & 53,95 & 216,81 & 43,36 & 72,46 & 96,54 & 19,31 & 88,97 & 31,49 & 6,30 \\
\hline Rala (VR) & 58,70 & 178,71 & 35,74 & 76,42 & 78,37 & 15,67 & 91,85 & 22,54 & 4,51 \\
\hline
\end{tabular}
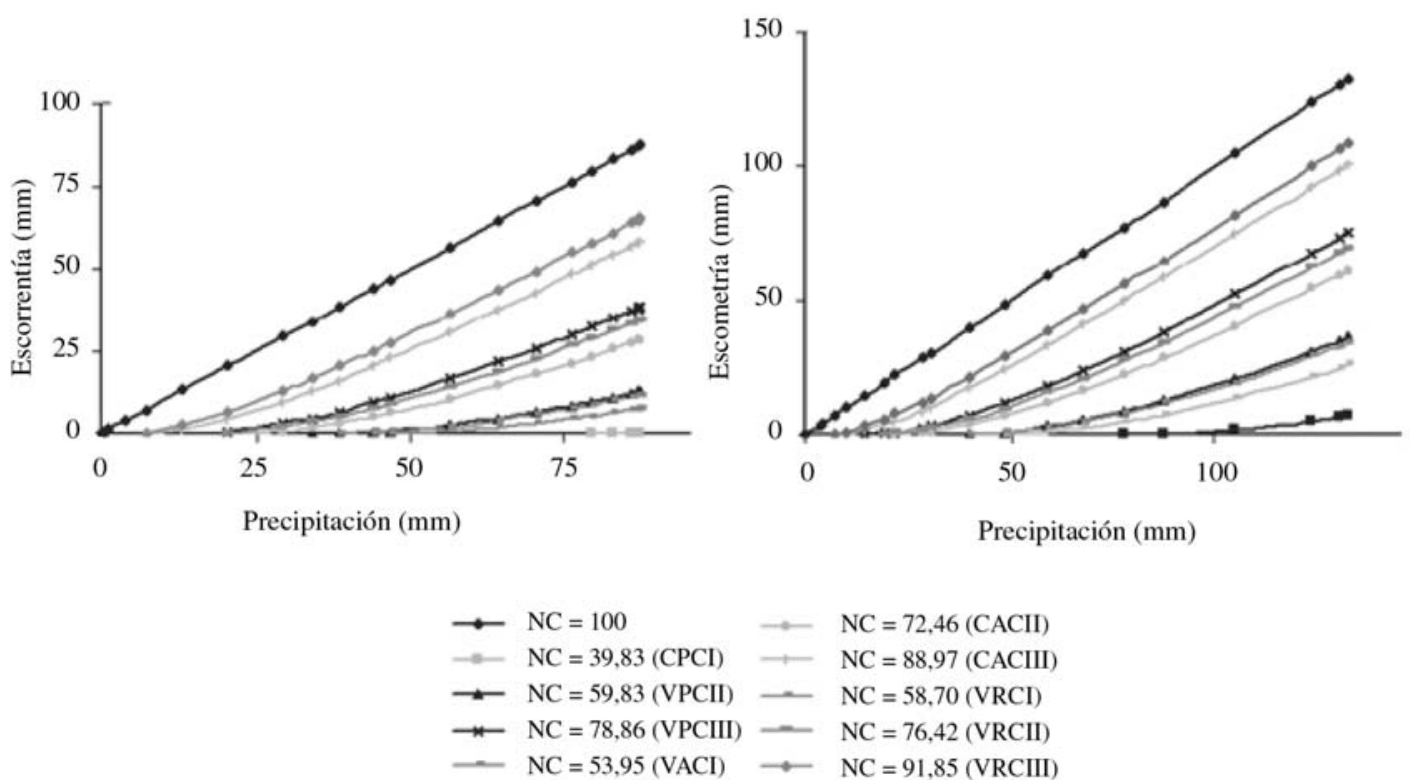

Figura 4. Escorrentía v/s precipitación y números de curva para la tormenta 5 y 13.

Runoff v/s rainfall and curve number for storm 5 and 13 .

\section{DISCUSIÓN}

Del análisis de las 30 tormentas se aprecia que, independientemente de las duraciones, la condición III genera los mayores coeficientes de escorrentía promedio, y la situación de vegetación plena provoca el efecto contrario. De esta forma, si el suelo está saturado y a una determinada hora la intensidad de precipitación es alta, el umbral crítico de escorrentía se supera rápidamente, contrario a lo que sucede en suelos secos o en situaciones de precipitaciones escasas. En cuanto a la vegetación, a medida que ésta es más densa, las escorrentías son menores, ya que juega un rol en la intercepción del agua caída, demorando la escorrentía superficial y favoreciendo la infiltración, lo que se condice con lo señalado por autores como Lindholm y Stembeck (1993), Farmer et al. (2003) y Pizarro et al. (2005). Esto queda de manifiesto al observar los cuadros 4 y 5 , que representan las máximas tormentas de alta y baja intensidad respectivamente y que muestran el comportamiento de los coeficientes de escorrentía durante las 24 horas de duración de las tormentas. En ellas se aprecian el desfase temporal y las diferencias de magnitud del coeficiente de escorrentía generado para cada tormenta, al estar sometido a diferentes condiciones de cobertura vegetal y humedad del suelo. Así, a mayor humedad del suelo y menor cobertura vegetal, el tiempo en sobrepasar el umbral crítico de escorrentía $\mathrm{I}_{\mathrm{o}}$ es menor y las precipitaciones efectivas generadas son mayores, determinando un coeficiente cercano a la unidad al terminar la tormenta. Por otro lado, si la presencia de vegetación es plena y la humedad precedente del suelo es menor, las precipitaciones efectivas son muy bajas en relación a las totales caídas, y el tiempo que transcurre para superar $\mathrm{I}_{\mathrm{o}}$ es notoriamente mayor.

De lo anterior se desprende que la metodología del número de curva ofrece una interesante perspectiva para 
Cuadro 4. Coeficiente de escorrentía para las tormentas de alta intensidad Runoff coefficients for high intensity storms.

\begin{tabular}{|c|c|c|c|c|c|c|c|c|c|c|c|c|c|c|c|c|}
\hline & & & & & & & Tor & entas & & & & & & & & \\
\hline Condición & horas & 1 & 2 & 3 & 4 & 5 & 6 & 7 & 8 & 9 & 10 & 11 & 12 & 13 & 14 & 15 \\
\hline & 1 & - & - & - & - & 0,04 & - & - & - & - & - & - & 0,12 & - & - & - \\
\hline VPCI & 2 & - & - & - & - & - & - & - & - & - & - & - & - & - & - & - \\
\hline & 4 & - & - & - & - & - & - & - & - & - & - & - & - & - & - & - \\
\hline & 1 & 0,15 & 0,19 & 0,16 & 0,21 & 0,27 & 0,14 & 0,21 & 0,22 & 0,08 & 0,13 & 0,11 & 0,14 & 0,35 & 0,26 & 0,14 \\
\hline VPCII & 2 & - & - & - & - & 0,07 & - & 0,02 & 0,01 & - & - & - & - & 0,17 & 0,03 & - \\
\hline & 4 & - & - & - & - & - & - & - & - & - & - & - & - & - & - & - \\
\hline & 1 & 0,37 & 0,40 & 0,38 & 0,52 & 0,59 & 0,44 & 0,43 & 0,43 & 0,32 & 0,40 & 0,45 & 0,41 & 0,53 & 0,59 & 0,42 \\
\hline VPCIII & 2 & 0,19 & 0,24 & 0,21 & 0,22 & 0,36 & 0,17 & 0,26 & 0,21 & 0,06 & 0,21 & 0,21 & 0,17 & 0,37 & 0,35 & 0,21 \\
\hline & 4 & 0,02 & 0,06 & 0,02 & 0,01 & 0,11 & 0,01 & 0,07 & 0,06 & - & - & 0,01 & 0,02 & 0,20 & 0,08 & - \\
\hline & 1 & 0,08 & 0,12 & 0,09 & 0,13 & 0,20 & 0,08 & 0,14 & 0,16 & 0,02 & 0,05 & 0,06 & 0,08 & 0,28 & 0,14 & 0,05 \\
\hline VACI & 2 & - & - & - & - & 0,01 & - & - & - & - & - & - & - & 0,08 & - & - \\
\hline & 4 & - & - & - & - & - & - & - & - & - & - & - & - & - & - & - \\
\hline & 1 & 0,29 & 0,35 & 0,33 & 0,42 & 0,45 & 0,33 & 0,39 & 0,32 & 0,18 & 0,29 & 0,33 & 0,29 & 0,47 & 0,50 & 0,34 \\
\hline VACII & 2 & 0,11 & 0,19 & 0,11 & 0,12 & 0,25 & 0,08 & 0,16 & 0,17 & 0,01 & 0,09 & 0,09 & 0,11 & 0,29 & 0,20 & 0,10 \\
\hline & 4 & - & - & - & - & 0,02 & - & - & - & - & - & - & - & 0,08 & - & - \\
\hline & 1 & 0,62 & 0,54 & 0,66 & 0,71 & 0,74 & 0,72 & 0,55 & 0,63 & 0,52 & 0,57 & 0,67 & 0,64 & 0,68 & 0,72 & 0,60 \\
\hline VACIII & 2 & 0,39 & 0,39 & 0,39 & 0,49 & 0,61 & 0,42 & 0,50 & 0,42 & 0,24 & 0,40 & 0,50 & 0,43 & 0,51 & 0,57 & 0,43 \\
\hline & 4 & 0,22 & 0,27 & 0,25 & 0,25 & 0,35 & 0,17 & 0,26 & 0,24 & 0,06 & 0,19 & 0,25 & 0,15 & 0,40 & 0,37 & 0,26 \\
\hline & 1 & 0,13 & 0,20 & 0,15 & 0,19 & 0,26 & 0,15 & 0,18 & 0,22 & 0,06 & 0,11 & 0,10 & 0,14 & 0,33 & 0,24 & 0,12 \\
\hline VRCI & 2 & - & 0,01 & - & - & 0,07 & - & 0,01 & - & - & - & - & - & 0,15 & 0,02 & - \\
\hline & 4 & - & - & - & - & - & - & - & - & - & - & - & - & - & - & - \\
\hline & 1 & 0,36 & 0,38 & 0,39 & 0,46 & 0,53 & 0,40 & 0,38 & 0,39 & 0,26 & 0,36 & 0,39 & 0,38 & 0,51 & 0,54 & 0,37 \\
\hline VRCII & 2 & 0,17 & 0,23 & 0,19 & 0,17 & 0,30 & 0,15 & 0,25 & 0,21 & 0,05 & 0,15 & $0,15-$ & $0,15-$ & 0,38 & 0,29 & 0,18 \\
\hline & 4 & - & 0,04 & - & - & 0,10 & - & 0,03 & 0,02 & - & - & - & - & 0,14 & 0,05 & - \\
\hline & 1 & 0,72 & 0,62 & 0,75 & 0,79 & 0,80 & 0,78 & 0,64 & 0,70 & 0,57 & 0,67 & 0,75 & 0,74 & 0,75 & 0,79 & 0,69 \\
\hline VRCIII & 2 & 0,77 & 0,47 & 0,51 & 0,53 & 0,73 & 0,54 & 0,46 & 0,54 & 0,37 & 0,53 & 0,57 & 0,56 & 0,71 & 0,68 & 0,55 \\
\hline & 4 & 0,29 & 0,31 & 0,30 & 0,38 & 0,48 & 0,27 & 0,39 & 0,32 & 0,16 & 0,32 & 0,33 & 0,26 & 0,40 & 0,38 & 0,38 \\
\hline
\end{tabular}

(-): Sin escorrentía superficial.

afrontar los estudios hidrológicos a partir de características físicas y geomorfológicas, en cuencas que carezcan de información fluviométrica. El estudio demostró que el comportamiento del coeficiente de escorrentía está determinado principalmente por tres factores: la cobertura de vegetación, la humedad del suelo y la intensidad de precipitación, siendo esta última la que más influye en su variación.

En cuanto a la cobertura vegetacional, se observó que los mayores coeficientes de escorrentía generados fueron para aquellas situaciones en donde la vegetación poseía una cobertura menor a $25 \%$, mientras que la condición de humedad del suelo, que presentó los coeficientes de escorrentía máximos, fue para una condición III, es decir, para un suelo saturado.
Por otra parte, con respecto a las regresiones para la estimación de $\mathrm{C}$, es importante señalar que para el primer escenario (VPCI) no se determinó un modelo de regresión, ya que ninguno resultó ser óptimo, debido a la poca cantidad de información obtenida para este caso, y ello derivado de que en ese escenario prácticamente no hubo escorrentía. Para los demás se puede apreciar la existencia de umbrales de escorrentía, determinados por los valores negativos de la constante del modelo (a), además de los valores bajo uno de la pendiente (b), lo que determina que deben producirse ciertos montos mínimos de precipitación en 24 horas para que se generen escorrentías. Según esto, la condición de humedad III es la que requeriría de menores montos de precipitación en 24 horas, los cuales varían entre 2,81 a 9,5 $\mathrm{mm}$, para las 
Cuadro 5. Coeficientes de escorrentía para tormentas de baja intensidad. Runoff coefficients for low intensity storms.

\begin{tabular}{cccccccccccccccccc}
\hline & \multicolumn{1}{c}{ Condición } & horas & 1 & 2 & 3 & 4 & 5 & 6 & 7 & 8 & 9 & 10 & 11 & 12 & 13 & 14 & 15 \\
\hline \multirow{2}{*}{ VPCII } & 1 & - & - & - & - & - & 0,10 & 0,02 & 0,15 & 0,03 & 0,09 & - & 0,03 & - & - & - \\
& 2 & - & - & - & - & - & - & - & - & - & - & - & - & - & - & - \\
& 4 & - & - & - & - & - & - & - & - & - & - & - & - & - & - & - \\
\multirow{2}{*}{ VACII } & 1 & - & - & - & - & - & 0,03 & - & 0,03 & - & 0,03 & - & - & - & - & - \\
& 2 & - & - & - & - & - & - & - & - & - & - & - & - & - & - & - \\
& 4 & - & - & - & - & - & - & - & - & - & - & - & - & - & - & - \\
\multirow{2}{*}{ VACIII } & 1 & - & 0,07 & 0,19 & 0,18 & 0,21 & 0,36 & 0,23 & 0,44 & 0,26 & 0,28 & 0,11 & 0,21 & 0,18 & 0,04 & 0,11 \\
& 2 & - & - & - & - & - & 0,13 & 0,05 & 0,18 & 0,06 & 0,08 & - & - & - & - & - \\
& 4 & - & - & - & - & - & - & - & - & - & - & - & - & - & - & - \\
\multirow{2}{*}{ VRCII } & 1 & - & - & - & - & - & 0,09 & - & 0,11 & 0,01 & 0,07 & - & - & - & - & - \\
& 2 & - & - & - & - & - & - & - & - & - & - & - & - & - & - & - \\
& 4 & - & - & - & - & - & - & - & - & - & - & - & - & - & - & - \\
\multirow{2}{*}{ VRCIII } & 1 & 0,06 & 0,17 & 0,27 & 0,29 & 0,20 & 0,51 & 0,30 & 0,52 & 0,34 & 0,38 & 0,20 & 0,31 & 0,32 & 0,11 & 0,22 \\
& 2 & - & - & 0,02 & 0,04 & 0,06 & 0,23 & 0,09 & 0,26 & 0,13 & 0,10 & 0,03 & 0,14 & 0,06 & - & - \\
& 4 & - & - & - & - & - & 0,04 & - & 0,04 & - & 0,03 & - & - & - & - & - \\
\hline
\end{tabular}

(-): sin escorrentía superficial.

Las condiciones VPCI, VPCIII, VACI y VRCI no presentaron escorrentía en ninguna de las ocasiones observadas.

Cuadro 6. Modelos seleccionados para la estimación del coeficiente de escorrentía.

Selected models for estimating runoff coefficient.

\begin{tabular}{|c|c|c|c|c|c|c|c|}
\hline Condición / modelo & Estimación & $\begin{array}{c}\text { Error } \\
\text { Estándar }\end{array}$ & Estadístico t & $\begin{array}{c}\text { Valor } \\
P\end{array}$ & $\begin{array}{l}\text { Durbin- } \\
\text { Watson }\end{array}$ & $\mathrm{R}^{2}$ & EEE \\
\hline \multicolumn{8}{|l|}{ VPCII $C=\alpha+\beta * P$} \\
\hline Intercepto & $-0,0697$ & 0,00861 & $-8,0915$ & 0,0000 & 1,57 & 0,9732 & 0,0285 \\
\hline Pendiente & 0,0005 & 0,00016 & 31,8234 & 0,0000 & & & \\
\hline \multicolumn{8}{|c|}{ VPCIII C $=\alpha+\beta *$ raíz (p) } \\
\hline Intercepto & $-0,3878$ & 0,02669 & $-14,5257$ & 0,0000 & 1,56 & 0,9699 & 0,057 \\
\hline Pendiente & 0,1262 & 0,00412 & 30,6022 & 0,0000 & & & \\
\hline \multicolumn{8}{|l|}{ VACI $C=\alpha+\beta * P$} \\
\hline Intercepto & $-0,0606$ & 0,01088 & $-5,56712$ & 0,0000 & 0,52 & 0,9158 & 0,036 \\
\hline Pendiente & 0,0037 & 0,00021 & 17,7849 & 0,0000 & & & \\
\hline \multicolumn{8}{|l|}{ VACII $C=\left(\alpha+\beta^{*} p\right)^{2}$} \\
\hline Intercepto & $-0,0459$ & 0,03767 & $-1,2202$ & 0,0000 & 1,73 & 0,8795 & 0,090 \\
\hline Pendiente & 0,0104 & 0,00071 & 14,5838 & 0,0000 & & & \\
\hline \multicolumn{8}{|c|}{ VACIII $C=\alpha+\beta^{*} \ln (\mathrm{P})$} \\
\hline Intercepto & $-0,5745$ & 0,03029 & $-18,9617$ & 0,0000 & 1,57 & 0,9819 & 0,031 \\
\hline Pendiente & 0,3442 & 0,00867 & 39,6968 & 0,0000 & & & \\
\hline \multicolumn{8}{|l|}{ VRCI $\mathrm{C}=\alpha+\beta * \mathrm{P}$} \\
\hline Intercepto & $-0,0681$ & 0,00850 & $-8,012169$ & 0,0000 & 1,66 & 0,9693 & 0,0281 \\
\hline Pendiente & 0,0049 & 0,00016 & 30,2789 & 0,0000 & & & \\
\hline \multicolumn{8}{|l|}{ VRCII $C=\left(\alpha+\beta^{*} p\right)^{2}$} \\
\hline Intercepto & $-0,0161$ & 0,04521 & $-0,356606$ & 0,0000 & 1,90 & 0,8433 & 0,149 \\
\hline Pendiente & 0,0108 & 0,00086 & 12,5346 & 0,0000 & & & \\
\hline \multicolumn{8}{|c|}{ VRCIII C $=\alpha+\beta^{*}$ raíz (p) } \\
\hline Intercepto & $-0,0799$ & 0,04507 & 1,7719 & 0,0000 & 1,57 & 0,8785 & 0,096 \\
\hline Pendiente & 0,1010 & 0,00696 & 14,5182 & 0,0000 & & & \\
\hline
\end{tabular}

$\alpha, \beta$ : parámetros de la regresión. 
condiciones de vegetación rala y plena, respectivamente. Para el caso de la otra condición extrema, la condición I, los valores de precipitación que generan escorrentía fluctúan entre los 14 y $16,5 \mathrm{~mm}$ para las condiciones de vegetación rala y actual, respectivamente.

En general, se aprecia que los modelos utilizados para determinar los coeficientes de escorrentía presentan una muy buena calidad de ajuste, lo que asegura una estimación y pronóstico del valor $\mathrm{C}$ en marcos confiables.

\section{CONCLUSIONES}

Según lo planteado en este estudio, la presencia y tipo de cubierta vegetal cumplen un rol importante en la reducción y/o retraso en la generación de escorrentías directas, independiente de las condiciones precedentes de humedad. Las condiciones precedentes de humedad del suelo y las intensidades de precipitación son las que determinan principalmente las cantidades de escorrentías sobre la cuenca, más que el tipo de cobertura vegetal que posea un área geográfica determinada. De lo anterior se desprende que una alta intensidad de precipitación define altos coeficientes de escorrentía, casi de forma independiente de la vegetación presente, ya que, si a la alta intensidad se asocia un suelo húmedo, los coeficientes serían cercanos a uno y para distintas situaciones vegetacionales.

\section{REFERENCIAS}

Calder I. 1992. Hydrologic effects of land-use change. In Maidment D ed. Handbook of Hydrology. Mc Graw-Hill, Estados Unidos. 1424 p.

Chow V, D Maidment, D Mays. 1994. Hidrología Aplicada. Santa Fe, Colombia. McGraw-Hill. 584 p.

Cid L, C Mora, M Valenzuela. 1990. Inferencia Estadística. Concepción, Chile. Universidad de Concepción. 319 p.

CIREN-CORFO (Centro de Información de Recursos Naturales, CL). 1983. Materiales y Símbolos. Proyecto Aerofotogramétrico CHILE/OEA/BID. Santiago, Chile. 53 p.

CONAF-CONAMA (Corporación Nacional Forestal, CL). 1997. Catastro y evaluación de los recursos vegetacionales nativos de Chile. Santiago, Chile. 126 p.

Dirección General de Aguas. 1987. Balance hídrico de Chile. Santiago, Chile. Dirección General de Aguas, Ministerio de Obras Públicas. 50 p.
Farmer D, M Sivapalan, C Jothity Angkoon. 2003. Climate, soil, and vegetation controls upon the variability of water balance in temperate and semiarid landscapes: Downward approach to water balance analysis. Water Resources Research 39(2):1-21.

Gujarati D. 1992. Econometría. D. F. México, México. Mc Graw-Hill Latinoamericana, 2a edición. 597 p.

Hibbert A. 1966. Forest treatment effects on water yield. In Proceedings of a National Science Foundation advanced science seminar, International symposium on forest hydrology. Pergamon Press, USA. 527-543 p.

Huber A, C Oyarzún. Precipitación neta e intercepción en un bosque adulto de Pinus radiata (D.Don). Bosque 5 (1):1320.

Iroumé A, A Huber. Intercepción de las lluvias por cubierta de bosques y efecto en los caudales de crecida en una cuenca experimental en Malalcahuello, IX Región, Chile. Bosque 21(1):45-56.

Lindholm P, I Stenbeck.1993. Rainfall, soil loss and the effect of vegetation cover; Results of measurements in a tree plantation area in West Pokot Districtof Kenya. International Rural Development Center. University of Agricultural Sciences. Working Paper $N^{\circ}$ 244. 25 p.

López F. 1998. Restauración hidrológica forestal y control de la erosión. Ingeniería Ambiental. Madrid, España. Tragsa, Tragsatec, Ministerio del Medio Ambiente, Ediciones Mundi-Prensa. 902 p.

Meunier M. La cubierta forestal y las crecidas en las pequeñas cuencas de montaña. Unasylva 47 (185):29-37.

Montgomery W, G Runger. 1996. Probabilidad y Estadísticas aplicadas a la Ingeniería. D.F. México, México. McGrawHill Latinoamericana. 895 p.

Oyarzún C, A Huber, S Vásquez. 1985. Balance hídrico en tres plantaciones de Pinus radiata I: Redistribución de las precipitaciones. Bosque 6 (1):3-14.

Pizarro R, A Benítez, C Farías, C Jordan, F Santibáñez, C Sangüesa, J Flores, E Martínez, L Román. Influencia de las masas boscosas en el régimen hídrico de una cuenca semiárida. Bosque 26(1):77-91.

Ponce V. 1989. Engineering Hydrology, Principles and Practices. New Jersey, USA, 640 p.

Reinhart K, A Eschner, G Trimble. 1963. Effects on streamflow of four forests practices in the mountains of West Virginia. U.S. Forest Service Research Paper, NE-1, Washington, USA. $79 \mathrm{p}$.

Stowhas L. 2003. Uso del método de la curva número en cuencas heterogéneas. XVI Congreso de Ingeniería Hidráulica. Pontificia Universidad Católica de Chile. Santiago, Chile.

Wright K, K Sendeck, R Rice, R Thomas. Logging effects on streamflow: Storm runoff at Caspar Creek in Northwestern California. Water Resources Research 26 (7):1657-1667. 